\title{
Small target detection using the Bilateral Filter based on Target Similarity Index
}

\author{
Tae-Wuk Bae, Sung-Hak Lee ${ }^{\text {a) }}$, and Kyu-Ik Sohng \\ School of Electrical Engineering and Computer Science, Kyungpook National \\ University, Buk-Gu, Daegu, 702-701, Korea \\ a)shak2@ee.knu.ac.kr
}

\begin{abstract}
This paper presents a new bilateral filter (BF) using the adaptive standard deviation based on the target similarity index (TSI) for small target detection. At first, the threshold value of pixel's TSI in window decides whether any pixel is a potential small target or not. The TSIs of the potential small target pixels are mapped to standard deviations of domain and range filters of the BF by linear mapping and the two standard deviations increase in proportion to the TSI for blurring the small targets. For the more blurred small targets, the filter size according to the TSI is increased. Experimental results demonstrate that the proposed method is more robust and efficient than the conventional methods.
\end{abstract}

Keywords: Small target, Bilateral Filter (BF), Target Similarity Index (TSI)

Classification: Microwave and millimeter wave devices, circuits, and systems

\section{References}

[1] A. D. Jong, "IRST and its perspective," Proc. SPIE, vol. 2552, pp. 206213, 1995.

[2] W. L. Wolfe, Introduction to Infrared System Design, SPIE Optical Engineering Press, Bellingham, WA, 1996.

[3] L. Chengjun, W. Ying, and S. Zeling, "A small target detection algorithm based on multi-scale energy cross," Proc. 2003 IEEE Int. Conf. Robot., Intelligent Syst. Signal Process., Changsha, China, vol. 2, pp. 1191-1196, Oct. 2003.

[4] T. Soni, R. Zeidler, and W. H. Ku, "Performance evaluation of 2D adaptive prediction filters for detection of small object in image data," IEEE Trans. Image Process., vol. 2, no. 3, pp. 327-340, July 1993.

[5] H. Sang, X. Shen, and C. Chen, "Architecture of a configurable 2D adaptive filter used for small object detection and digital image processing," Opt. Eng., vol. 42, no. 8, pp. 2182-2189, 2003.

[6] J. Barnett, "Statistical analysis of median subtraction filtering with application to point target detection in infrared backgrounds," Proc. SPIE, vol. 1050, pp. 10-18, 1989.

[7] Y. Xiong, et al., "An extended track-before-detect algorithm for infrared target detection," IEEE Trans. Aerosp. Electron. Syst., vol. 33, no. 3, pp. 1087-1092, 1997. 
[8] C. Tomasi and R. Manduchi, "Bilateral filtering for gray and color images," Proc. Int. Conf. Comput. Vis., pp. 839-846, 1998.

[9] C. I. Hilliard, "Selection of a clutter rejection algorithm for real-time target detection from an airborne platform," Proc. SPIE, vol. 4048, pp. 74-84, 2000 .

\section{Introduction}

In IR warning systems (IRWS) and IR search and tracking systems (IRST), the automatic detection and tracking of small targets is necessary for military applications to warn the moving small targets such as aircrafts and helicopters of enemy from a distance $[1,2,3]$. In past decades, many techniques have been proposed for suppressing the clutter and enhancing the detect-ability of small targets. Soni et al. have evaluated the performance of the two-dimensional least mean square (TDLMS) adaptive filter in target detection [4]. Sang et al. have enhanced the target detection method of two-dimensional normalized LMS (TDNLMS) filter [5]. Barnett has presented a median subtraction filter for point target detection [6]. But these techniques don't give the good performance in low contrast images having the dim small targets.

In this paper, we propose a new small target detection method using the $\mathrm{BF}$ based on the target similarity index (TSI). To operate the BF as the background predictor, we use the adaptive standard deviation adjusted by the TSI in the background and target region. So the proposed BF estimates the background region and blurs the target region.

\section{Bilateral Filter and its application in small target detection}

Tomasi and Manduchi proposed the BF as a nonlinear filter that smoothes the noise while preserving edges [8]. The predicted pixel by the BF is given by

$$
Y(i, j)=\sum_{l=-N}^{N} \sum_{k=-N}^{N} H(i, j ; l, k) X(l, k)
$$

where $Y(i, j)$ is the output image, $H(i, j ; l, k)$ is the nonlinear combination between the pixel $(l, k)$ in the window and the center pixel $(i, j)$ of the window and $X(l, k)$ is the degraded image (the original image). The nonlinear combination in the window is given by

$$
\begin{aligned}
& H(i, j ; l, k)= \\
& \begin{cases}w_{i . j}^{-1} \exp \left(-\frac{(l-i)^{2}+(k-j)^{2}}{2 \sigma_{d}^{2}}\right) \times \exp \left(\frac{(X(l, k)-X(i-j))^{2}}{2 \sigma_{r}^{2}}\right), & \text { if }(l, k) \in \Lambda_{i, j} \\
0 & \text { otherwise }\end{cases}
\end{aligned}
$$

where $\Lambda_{i, j}=\{(l, k):(l, k) \in(i-N, i+N) \times(j-N, j+N)\}$ represents the pixels belong to the window, $\sigma_{d}$ and $\sigma_{r}$ are the standard deviations of the 
domain and range filters and $w_{i, j}$ is a normalization factor given by

$$
w_{i, j}=\sum_{l=i-N}^{i+N} \sum_{k=j-N}^{j+N} \exp \left(-\frac{(l-i)^{2}+(k-j)^{2}}{2 \sigma_{d}^{2}}\right) \times \exp \left(-\frac{(X(l, k)-X(i-j))^{2}}{2 \sigma_{r}^{2}}\right)
$$

It assures that the filter preserves the local region having constant gray levels in the image.

The BF adopts two low-pass Gaussian filters for the domain and range filters. The domain low-pass Gaussian filter (D-LPGF) gives higher weight to pixels that are spatially closer to the center pixel. The range low-pass Gaussian filter (R-LPGF) gives higher weight to pixels that have more similar value to the center pixel's value in gray scale.

To apply the BF to small target detection, the effect of $\sigma_{d}$ and $\sigma_{r}$ should be considered. $\sigma_{d}$ controls the width of the D-LPGF. For a fixed $\sigma_{r}$, the small $\sigma_{d}$ makes the limited smoothing but the large $\sigma_{d}$ makes the strong smoothing in the window. On the other, $\sigma_{r}$ controls the width of the R-LPGF and determines the range of application when smoothing pixels to the average level. If $\sigma_{r}$ is larger than the pixel range in the window, the R-LPGF will assign the similar weight to every pixel in the range. But, it doesn't have an effect on the overall $\mathrm{BF}$. In the small target region, a small $\sigma_{r}$ will make the R-LPGF a dominant smoothing filter in the BF.

For the exact prediction of the background region, the BF set the higher weight to the center pixel in D-LPGF and to the pixels having a similar value to the center pixel in R-LPGF. To do it, $\sigma_{d}$ and $\sigma_{r}$ should have relatively small values in the background region. On the contrary, $\sigma_{d}$ and $\sigma_{r}$ should have relatively large values to blur the target region. Because a larger $\sigma_{d}$ and $\sigma_{r}$ make the width of the D-LPGF and R-LPGF more extensive, so it can blur the target region.

\section{Bilateral Filter based on Target Similarity Index}

The procedure of the proposed $\mathrm{BF}$ is as the following. At first, we calculate the TSI of a center pixel in the window. Then the proposed BF operation is decided through the background discrimination by the TSI. Because the TSI of background pixel has small value, these pixels are not filtered by the proposed BF. For potential target pixels, the TSI of the pixel decides the standard deviations and filter size. The predicted background image is subtracted from an original image, in which small targets can be detected [7].

\subsection{TSI}

The proposed algorithm calculates the background similarity index (BSI) using the random variable of the background region. It is supposed that a random variable of the window is $x=\left\{x_{i} \mid i=1,2, \ldots, P\right\}$ and a random variable of the background region is $y=\left\{y_{i} \mid i=1,2, \ldots, P\right\}$, then the these two random variable sets have the same distribution characteristics. $P$ (i.e. the value of 25) is the number of the respective samples because the basic 
filter size is $5 \times 5$. The BSI, $\varphi$ is given by

$$
\varphi=\frac{2 \overline{x y}}{(\bar{x})^{2}+(\bar{y})^{2}} \cdot \frac{2 \sigma_{x} \sigma_{y}}{\left(\sigma_{x}^{2}+\sigma_{y}^{2}\right)}
$$

where $\bar{x}, \sigma_{x}^{2}$ are the average and variance of $x$ and $\bar{y}, \sigma_{y}^{2}$ are the average and variance of $y$ and the dynamic range of $\varphi$ is $[0,1] . \varphi$ is modeled by the combination of the luminance ratio (LR) and contrast ratio (CR). In an Eq. (4), the first component represents the LR of the two random variables and it means the similarity of the average luminance. In case of $\bar{x}=\bar{y}, \mathrm{LR}$ is 1 . The second component means the similarity of CR between the window and the background, $\sigma_{x}^{2}$ and $\sigma_{y}^{2}$ represents the level of CR. In case of $\sigma_{x}^{2}=\sigma_{y}^{2}$, CR is 1 . If $\varphi$ is close to 1 , it means that the background and the window has the similar distribution characteristics. On the other hand, if $\varphi$ is close to 0 , it means that the window is included to the potential target region. So the TSI can be calculated as a reciprocal of the BSI. The TSI, $\tau$ is given by

$$
\tau=(1 / \varphi)=\frac{(\bar{x})^{2}+(\bar{y})^{2}}{2 \overline{x y}} \cdot \frac{\left(\sigma_{x}^{2}+\sigma_{y}^{2}\right)}{2 \sigma_{x} \sigma_{y}}
$$

In the proposed algorithm, we use a linear mapping from $\left[n \tau_{\min }, n \tau_{\max }\right]$ to $\left[\sigma_{d \_ \text {min }}, \sigma_{d \_ \text {max }}\right],\left[\sigma_{r \_ \text {min }}, \sigma_{r \_ \text {max }}\right]$ when $n \tau(i, j)$ is the normalization of $\tau(i, j)$ at a pixel $(i, j) . n \tau_{\min }$ and $n \tau_{\max }$ are the minimum and maximum values in $n \tau(i, j)$. Now the proposed BF has the adaptive $\sigma_{d}$ and $\sigma_{r}$, which distinguishes the small target region from the background region.

\subsection{Adaptive filter size based on the TSI}

In the proposed BF, Blurring the target is a key technique for the background prediction. However, the flat region such as a constant background and an inner cloud must not be blurred. Because these regions have very low TSI, these pixels are excluded in the process of background prediction. And this scheme reduces the computation burden of the BF. The conditional formula to decide predicted background pixel at any pixel position is given by

$$
I(i, j)= \begin{cases}\tilde{X}(i, j), & \text { if }\left(n \tau(i, j) \geq \lambda \times n \tau_{\max }\right) \\ X(i, j), & \text { otherwise }\end{cases}
$$

where $\lambda$ is the weighting of $n \tau_{\max }$ for controlling the range of the BF application and it was set by 0.25 experimentally.

If any pixel satisfies the processing condition in Eq. (6), the predicted background pixel is substituted by $\tilde{X}(i, j)$ processed by the proposed BF for potential target pixel. Otherwise, original pixel, $X(i, j)$ is used for the background pixel.

To blur the target more, the filter size should be increased based on the TSI. To adjust the filter size $(2 M+1)(2 M+1)$ of the proposed BF, two thresholds are given by

$$
\begin{cases}M=N+2, & \text { if } n \tau(i, j)>\alpha \times n \tau_{\max } \\ M=N+1, & \text { else if } n \tau(i, j)>\beta \times n \tau_{\max }, \\ M=N, & \text { otherwise }\end{cases}
$$


where $\alpha=0.75$ and $\beta=0.5$ are the weighting of $n \tau_{\max }$ for controlling the filter size. These values are decided experimentally. $\alpha$ and $\beta$ is set in proportion to the $n \tau_{\max }$. Because the target region and cloud boundary region have the high TSI in comparison with the constant background region, the filter size becomes to be larger, so blur those regions more and more.

\section{Simulation results}

Fig. 1 shows the detection results for 4 test images using the proposed BF. The images have small targets in the constant background and cloud ( 8 bitdepth and $360 \times 240$ images $)$. We set $N=2(5 \times 5$ filter size $)$ for the initial filter size and $\sigma_{d \_ \text {min }}=0, \sigma_{d \_ \text {max }}=127, \sigma_{r \_ \text {min }}=0$, and $\sigma_{r \_ \text {max }}=255$ for the minimum and maximum standard deviation of the domain and range filters. $\sigma_{d \_ \text {max }}=127$ and $\sigma_{r \_ \text {max }}=255$ are set values for maximum blurring in the window of the BF for 8 bit IR images. Fig. 1 (a) shows original images containing small targets with different backgrounds. Fig. 1 (b) shows the predicted background images and Fig. 1 (c) shows the detection results. We get the detection results by subtracting the predicted background image from the original one, and then binarize the difference image by setting the threshold as $0.8 S_{\max }$, where $S_{\max }$ is the maximum grayscale in difference image. To make the targets even easier to distinguish, we modified the difference image with an exponential function given by

$$
M(i, j)=\exp \left(S(i, j) / \sigma^{2}\right)
$$

where $S(i, j)$ is the difference image. We set $\sigma=4$ experimentally, which makes small targets more prominent than the background clutters. Fig. 1 (d)

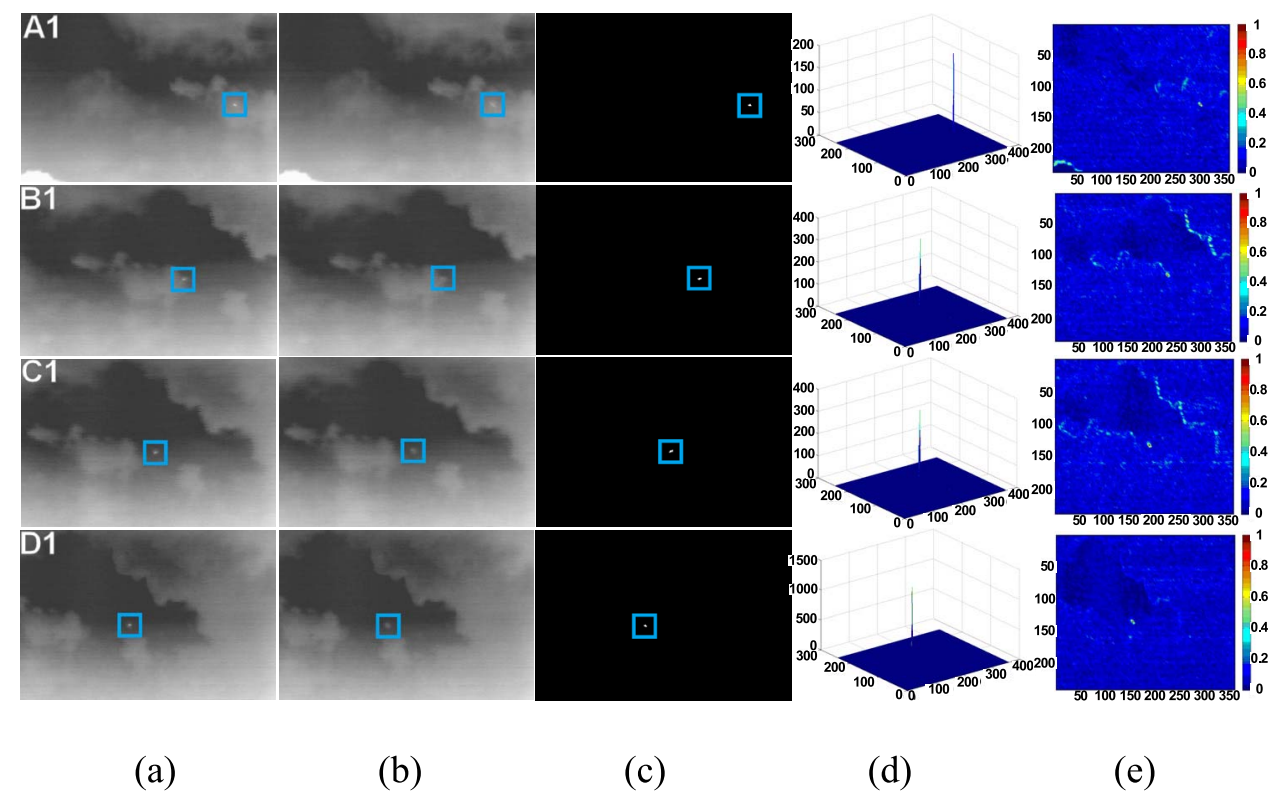

Fig. 1. Detection results for the 4 images within small targets. (a) Original images, (b) Predicted background images, (c) Detection results, (d) Intensity of the mapped image, and (e) TSI plots. 
shows the intensity of the mapped image. Fig. 1 (e) shows the TSI over the whole images.

Fig. 2 shows a subjective comparison between the conventional filters and the proposed method for D1 image. For objective comparison, two metrics, the signal-to-clutter ratio gain (SCRG) and the background suppression factor (BSF) [9], are employed. These are defined as

$$
S C R G=\frac{(S / C)_{\text {out }}}{(S / C)_{\text {in }}}, \quad B S F=\frac{C_{\text {in }}}{C_{\text {out }}}
$$

where $S$ is the signal level and $C$ is the clutter standard deviation in a normalized difference image. The experimental data using A1, B1, C1, and D1 are showed in Fig. 3. From the subjective and objective comparison results, we can conclude that the proposed BF shows better detection performance for IR small targets.

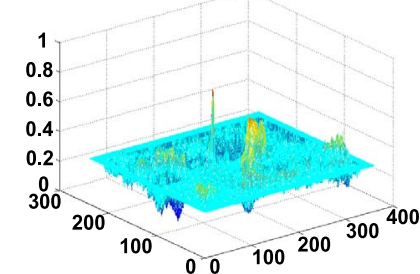

(a)

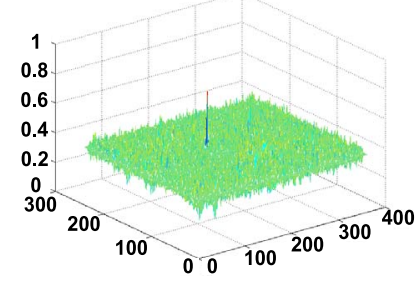

(d)

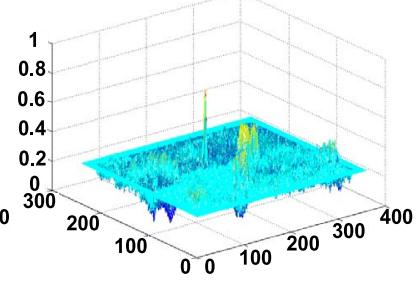

(b)

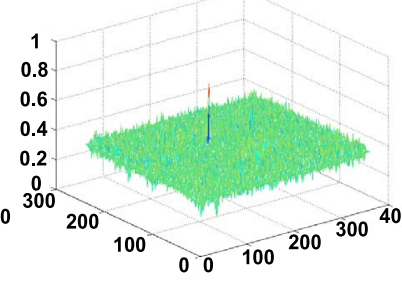

(e)

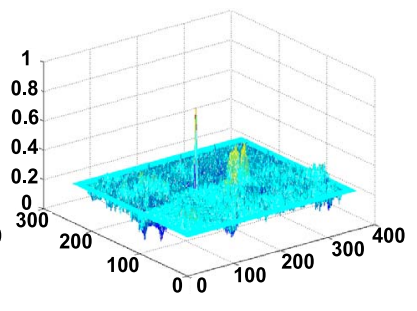

(c)

Fig. 2. Subjective comparison. (a) Median $11 \times 11$, (b) Median $9 \times 9$, (c) Median $7 \times 7$, (d) TDLMS $\left(u(\right.$ step size $\left.)=10^{-6}\right),\left(\right.$ e) TDNLMS $\left(u=11^{-7}\right)$, and (f) The proposed BF.

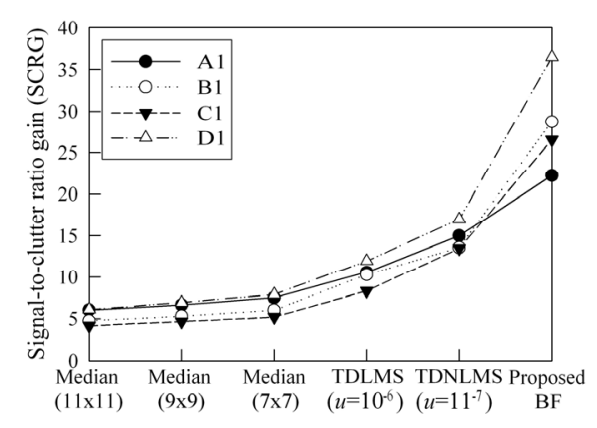

(a)

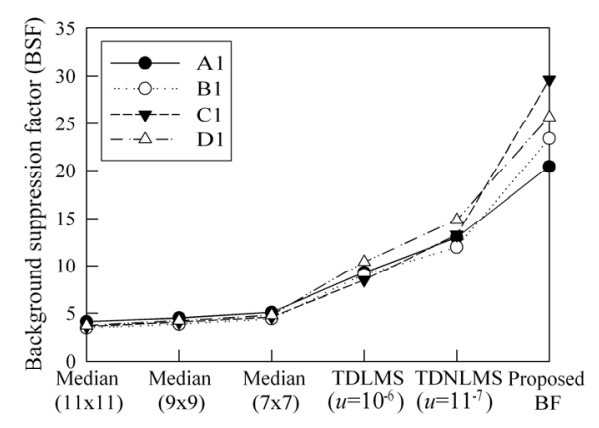

(b) 


\section{Conclusions}

The TSI of the original image decides the feature of the domain filter and range filter of the $\mathrm{BF}$. When the $\mathrm{BF}$ comes to a target region, the standard deviations of the proposed $\mathrm{BF}$ have the high value for the target blurred by TSI. To blur the target more, the filter size should be changed. We can be sure that the proposed $\mathrm{BF}$ is more suitable in the field of small target detection than the conventional methods. 